\title{
The membranous labyrinth in baculovirus- infected crustacean cells: possible roles in viral reproduction
}

\author{
John A. Couch \\ U.S. Environmental Protection Agency, Environmental Research Laboratory Gulf Breeze, Florida 32561, USA
}

\begin{abstract}
The origins and morphogenesis of the ninembranous labyrinth (ML) in Baculovirus penaei (BP) infected cells of penaeid shrimps (Crustacea:Decapoda) are described. The ML is usually a highly ordered, complex system of membranes arranged as a labyrinth or in concentric form in the cytoplasm near or against the nuclear envelope. The ML originates from dilated Golgi and endoplasmic reticulum vesicles, and from the outer nuclear envelope. It grows apparently from proliferation of the cellular membranes of these systems, and its development may be correlated with the stages of BP infection. It is hypothesized that, because of the close parallel and concurrent development of the ML and virus reproduction, and other evidence, the ML is virus-induced and-controlled and may play at least 3 roles in the virus reproductive strategy: (1) provides a conduit or transport system for viral precursors from cytoplasm to nucleus; (2) provides increased membrane surface and volume for increased ATPase activity (related to energy demand for virus reproduction and transport of viral products); and (3) provides a mechanism for cell collapse and release of virus and occlusion bodies at the end of virus reproduction period. Possible experimental methods with which to determine the functional role of the ML are discussed and implications for such a systern for nuclear, DNA, viruses are considered.
\end{abstract}

\section{INTRODUCTION}

The role of virally modified host cell structure in the reproductive strategy of viruses in not well understood. Many cytopathic effects in virus-infected cell systems have been considered merely a degenerative process following irreversible viral injury to the cell. In regard to this, Penman (1986) stated 'Indeed, the virus-specific cytopathic effect may very well be due not to gratuitous damage but, rather to specific reorganization of skeletal elements for the purpose of virus growth. Penman further points out that little attention has been given to the cellular, topographic aspects of viral behavior and such questions as transport of viral products between cell compartments (Penman 1986).

In 1974, a unique baculovirus of Crustacea was described for the first time (Couch 1974). This virus was named Baculovirus penaei (BP) because its hosts were marine penaeid shrimps. Apart from being the first occluded baculovirus described from a taxonomic group of hosts outside Insecta (Matthews 1982), the Baculovirus/crustacean host cell system possessed not only characteristics of other Baculovirus/host cell systems but several special and perhaps exaggerated characteristics to and of these systems thát may permit more insight into the possible role(s) of viral modified cellular structure in nuclear, DNA virus reproduction.

Chief among these new and/or exaggerated features in the Baculovirus/crustacean host cell system was the development of a highly organized membranous system in the cytoplasm of infected cells. Couch (1974, 1989) suggested the term 'membranous labyrinth' (ML) to denote this conspicuous membranous system whose appearance, proliferation, and development coincided with stages of virus infection, reproduction, assembly and final liberation of both free and occluded virus from crustacean host cells. The purpose of this paper is: (1) to describe, in some detail, the origin and morphogenesis of the ML in BP-infected crustacean cells; (2) to hypothesize several possible functions of the ML in reproduction and fate of $\mathrm{BP}$; and (3) to discuss the implications of these hypotheses for aiding in elucidating nuclear, DNA virus reproductive and cellular transport mechanisms generally. 


\section{MATERIALS AND METHODS}

Virus-infected tissues. All of the ultrastructural observations were made on tissue from naturally infected penaeid shrimps obtained from various sites in the Gulf of Mexico Host species were Penaeus duoraruan (pink shrimp), $P$ aztecus (brown shrimp). and $P$. setiterus (white shrimp).

BP infects cells of digestive gland epithelia, therefore, these glands were removed from shrimp, tissue

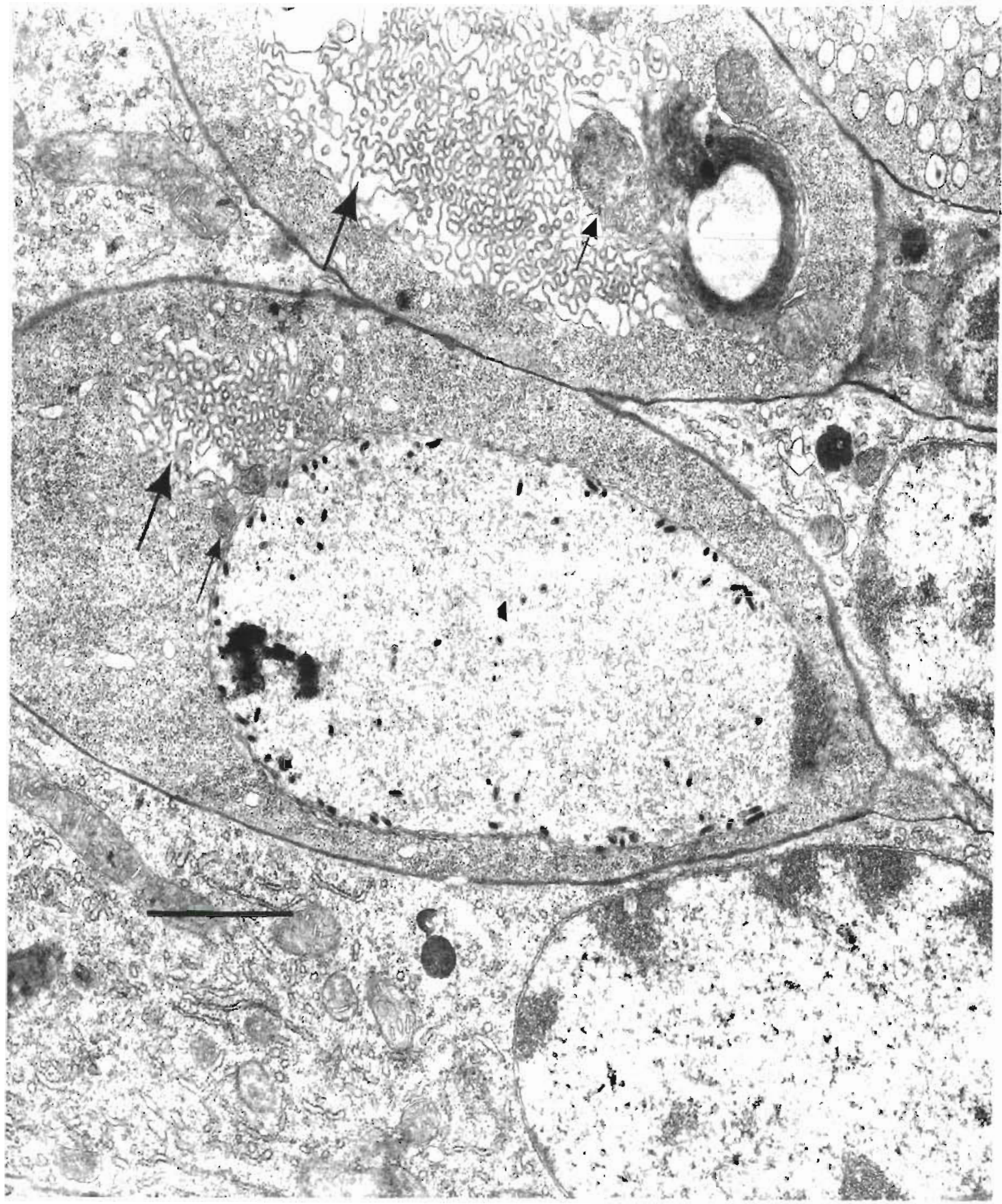

Fig 1 Early to intermediate stage of BP infection (Stage $C$ in Table 1) in digestive gland epithelial cells; note the relative sizes and nuclear proxumity of the ML (large arrows), heterochromatin diminution, virions in nucleus, many free ribosomes, and few remaining mitochondria associated only with the ML (small errows). Bar $=2 \mu \mathrm{m}$ 


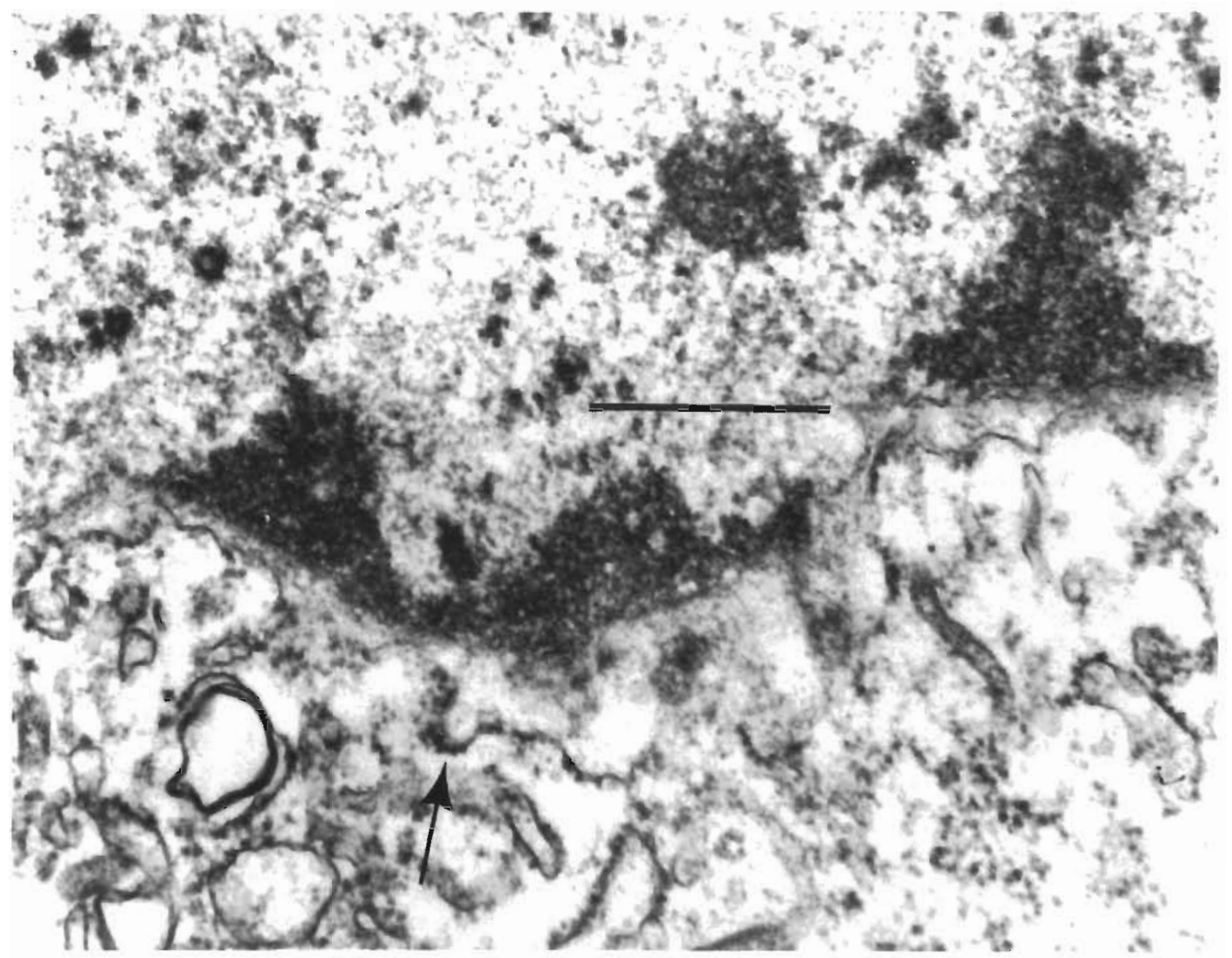

Fig. 2. Very early stage (B in Table 1) of BP infection; outer membrane of nuclear envelope outfolding (arrow), and heterochromatic marginating; virions not yet present. $\mathrm{Bar}=2 ! \mathrm{m}$

Table 1. Summary of presumed chronological concurrence of origin and morphogenesis of the ML and production, assembly, and maturation of both BP virions and occlusion bodies (OB). Compare with Stages A to E in Fig. 7

Stages in virus reproduction

A Virion uptake and translocation to nuclear pore. Virion uncoating at nuclear pore

B Granular and fibrillar virogenic stromata in nucleus

C Virogenic stromata and assembled virions and envelope membranes in nucleus

D Mature virions in nucleus; OB's forming, and occluding enveloped virions

E Virions and $O B$ 's cell free, and in remnants of host cell
Origin of ML and cellular changes

None

Heterochromatin diminishes; outer nuclear membrane folds outward; Golgi and ER vesicles dilate; detachment of polyribosomes from nuclear membrane and $E R$

Heterochromatin disappears; early ML grows in regions of Golgi and outer nuclear envelope via membrane proliferation; numerous unattached ribosomes (monosomes) appear in cytoplasm otherwise devoid of most normal organelles

ML at maximum size and organization; numerous 20 to $25 \mathrm{~nm}$ size particles in nucleus that appears to form paracrystalline structure of $O B$; dark amorphous material in cisternae of ML; mitocondria intimately associated with ML

ML unfolded and degenerate; cell lysis and destruction 
squashes were made of aliquots, and these exammed to determine presence or absence of characteristic tetrahedral occlusion bodies in coll nuclei indicating patent viral infections (Couch 1974)

Light microscopy. Viral-infected tissues were fixed in an FAA-filtered seawater fixatıve (Davidson's fixative), and degree and stages of cellular infection were determined by light microscopic examination. Histology sections 5 to $6 \mu \mathrm{m}$ thick were used. Histologic sections were stained with Harris hematoxylin.
Electron microscopy. For ultrastructural studies, digestive glands were diced in $2.5 \%$ glutaraldehyde for $30 \mathrm{~min}$ and postfixed in $1 \% \mathrm{OsO}_{4}$ for $30 \mathrm{~min}$ at 0 to $4{ }^{\circ} \mathrm{C}$ Fixed tissues were processed and finally embedded in Epon 812 according to the method of Hayat \& Giaquinta (1970) Sections 40 to $70 \mathrm{~nm}$ thick were collected on 300-mesh copper grids, and stained with uranyl acetate and lead citrate. Several hundred cell profiles were studied and photographed with a Zeiss EM9S2 or Zelss EM10 electron microscope.

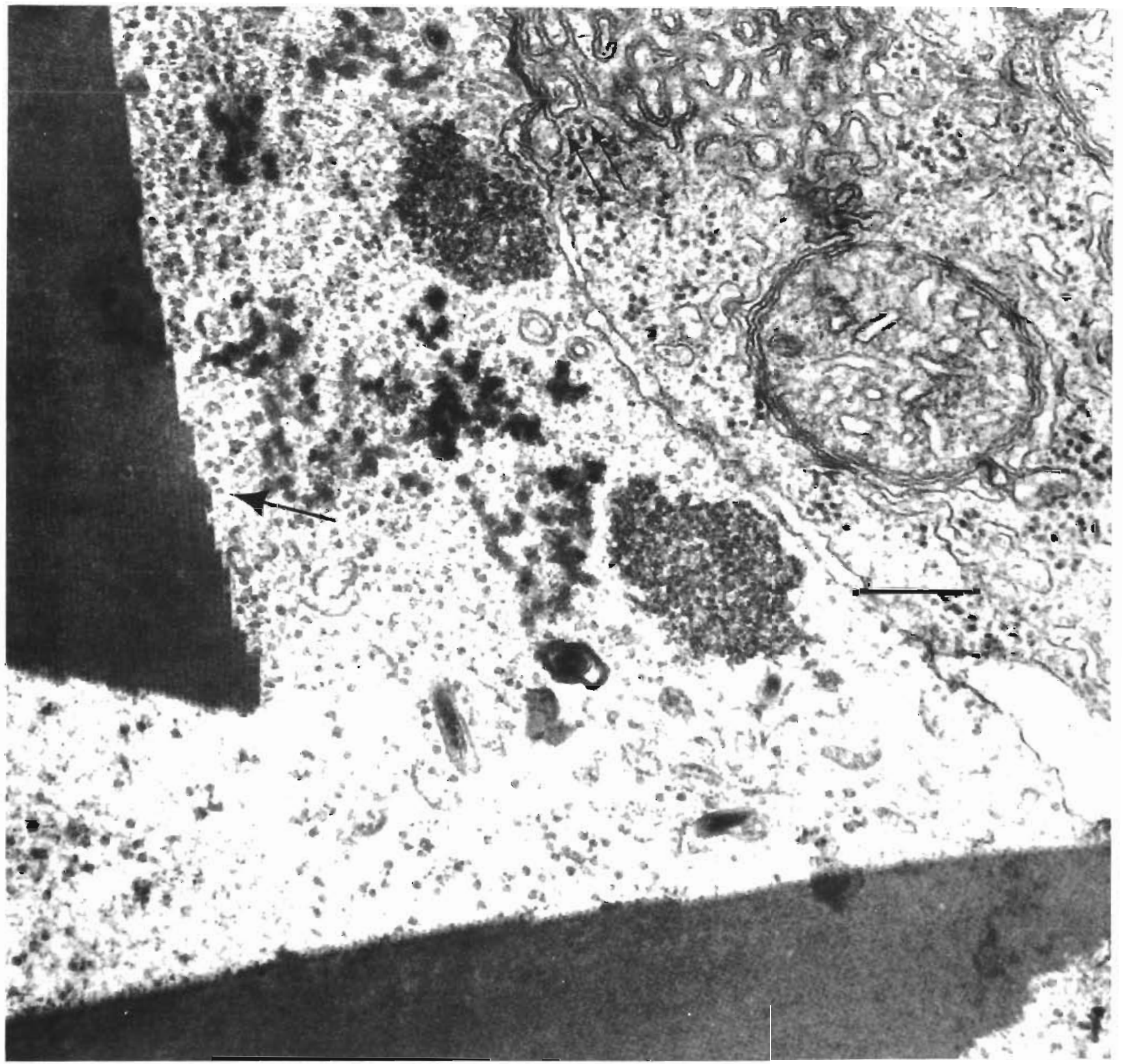

Fig. 3. The fundamental ML structure consists of 2 tightly opposed membranes with small band of cytoplasm sandwiched between (small arrows); note close association with nuclear envelope. Note, also, spherical particles (ca $20 \mathrm{~nm}$ ) of polyhedrin adjacent to growing lattice of $O B$ (large arrow). Bar $=500 \mathrm{~nm}$ 


\section{RESULTS}

Origin and morphogenesis of membranous labyrinth (ML) in host cells

The ML, found in penaeid shrimp digestive gland cells infected with BP, is a system of cellular membranes of unusual form and arrangement (Fig. 1). One special feature of this system of membranes is its apparent proliferative origin from a least 3 sources in BP-infected cells: the outer nuclear envelope, the Golgi, and the endoplasmic reticulum (ER) (Figs. 2 to 5). Another unique feature is the basic structure and relationships of the membranes themselves. Two separate, cellular membranes arose from pre-existing cellular membranes of the outer nuclear envelope. Golgi or ER, became tightly opposed to one another and grew to form either concentric (myelinoid) mem-

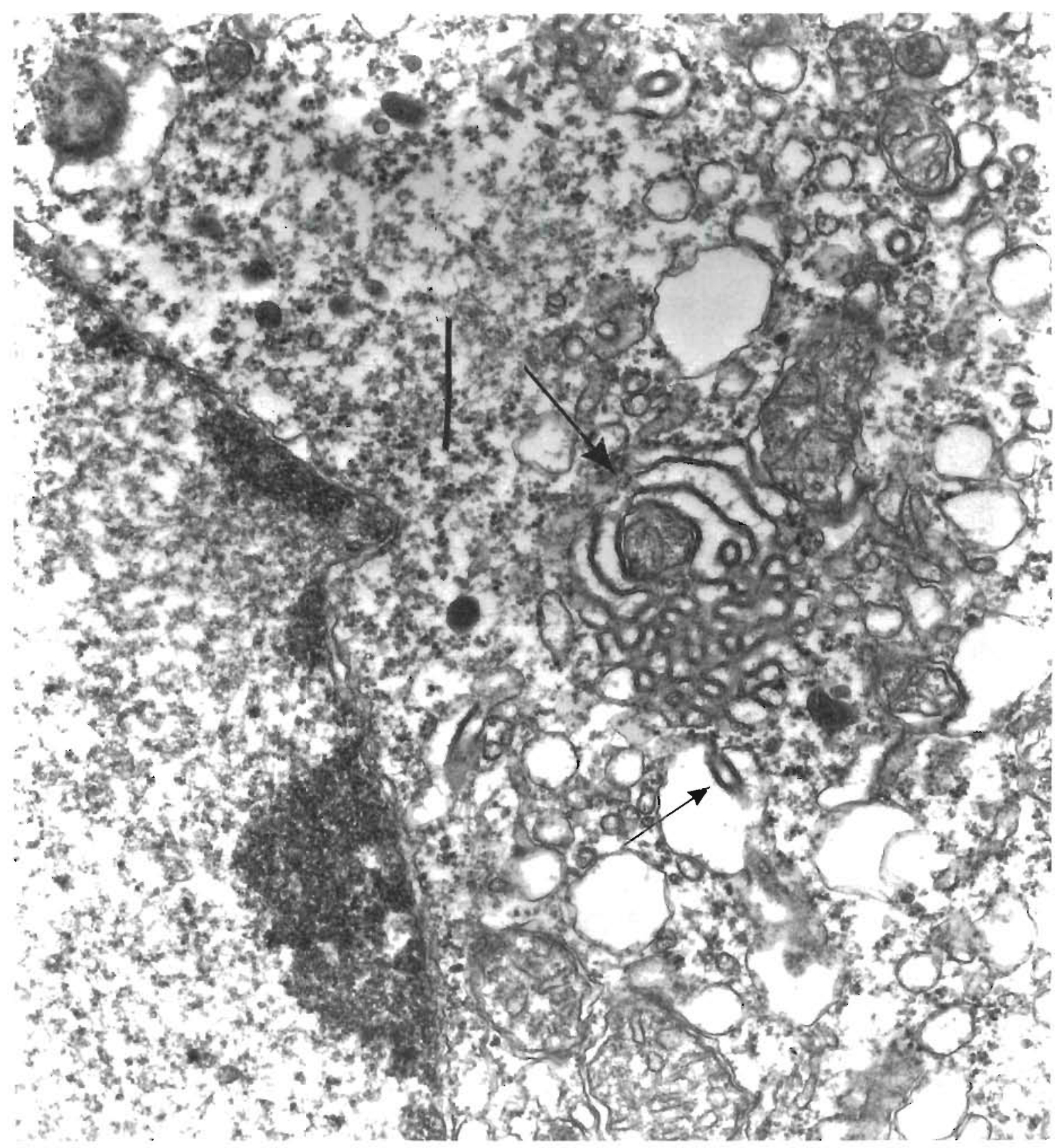

Fig. 4. Early development of ML in dilated Golgi near nucleus of BP-infected coll (large arrow); note the very earliest invagination of Golgi membrane into Golgi vesicle (small arrow); mitochondron is being unveloped by growing ML membranes. Bar $=625 \mathrm{~nm}$ 
brane masses or more highly organued labyrnthian structures near the nucleus of an infected cell (Figs. 3 and 6) Indeed, at low magnification the dual opposed membranes appeared to be a single structure of ca 10 to $20 \mathrm{~nm}$ in width (Fig. 1), but at higher magnification it was apparent that 2 tightly opposed. separate membranes $(40$ to $7.0 \mathrm{~nm}$ thick $)$ with a small cytoplasmic space between constituted the structure (Figs. 3 and 6)

The ML appeared in infected cells only; there has been a $100 \%$ association of the characteristic ML with infected cells, e.g. presence of virogenic stroma in nucleus, forming virions or formed, enveloped virions, and in later stages of ML development occlusion body and virions The concurrent development of the ML with virus and occlusion bodies (OBs) and other cytopathic effects will be described later in order to suggest a functional/developmental relationship between the two. The ML has never been associated with any other causative phenomenon in the host cells, 1.e. the ML is pathognomonic of BP infections

One major origination site for the $\mathrm{ML}$ is in dilated Golgi vesicles in the cytoplasm near the outer nuclear envelope membrane (Figs. 4 and 5). Incipient ML formation may begin in several of these vesicles by membranes budding into the vesicle (Figs, 4 and $7 \mathrm{C}$ ).

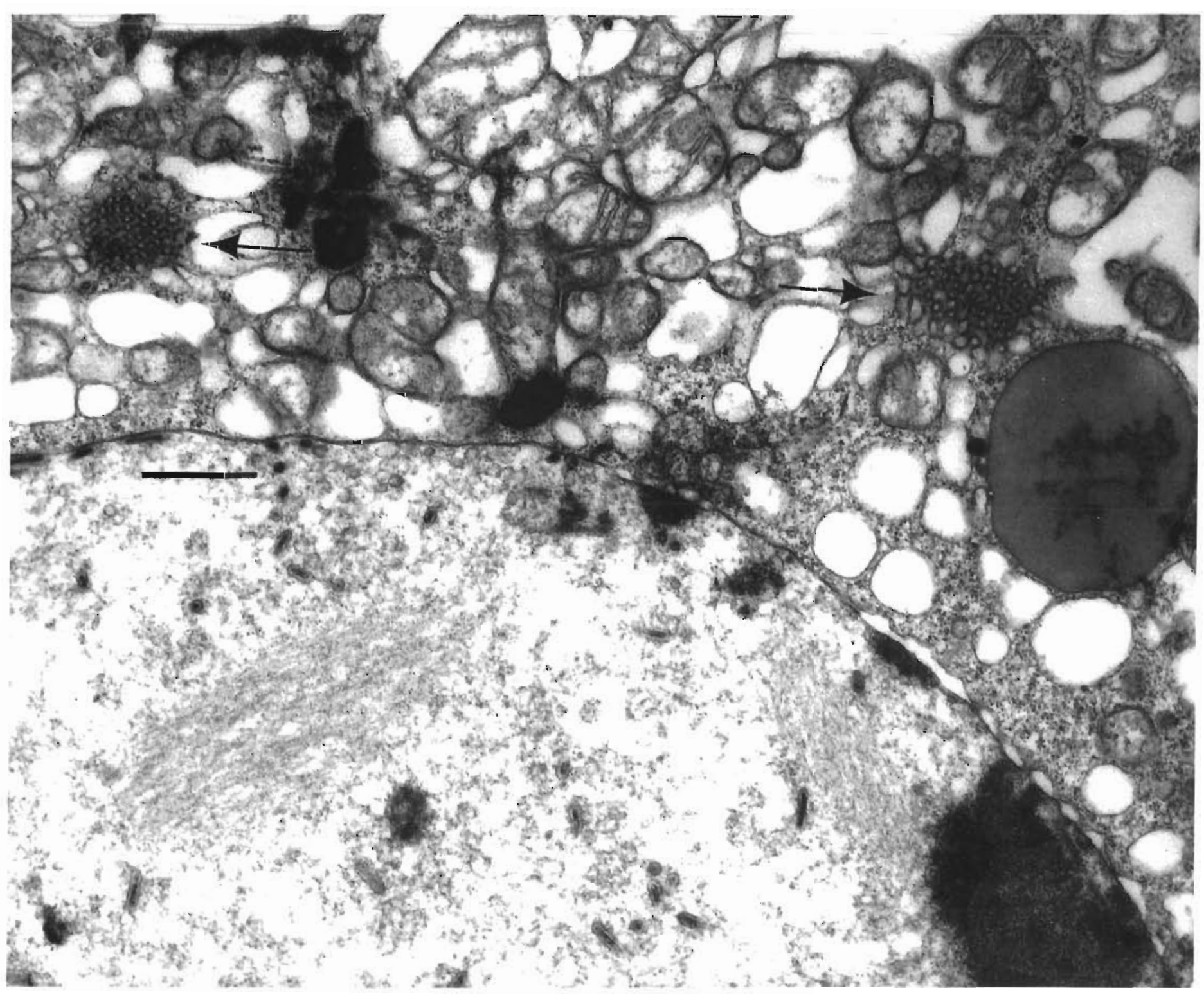

Fig 5 Small, early foci of ML associated with greatly dilated Golgi vesicles near nuclear envelope of Stage B-C, BP-infected cell, note unusual, branched mitochondrion between the 2 foci; grarular and fibrillar virogenic stromata and virions are found in the nucleus. Bar = $1 \mathrm{um}$ 
These ML systems may fuse as they grow larger, and ultimately most associate themselves with the outer nuclear envelope of the infected cell.

Based on my observations of many profiles of infected cells, another major site of origin of both myelinoid and labyrinthian forms of the ML has been at the outer nuclear envelope membrane (Figs. 2,3,7 and 8) or so near the nuclear envelope as to make resolution of the ML and nuclear envelope impossible. A semidiagrammatic depiction of the presumed morphogenetic sequence for the ML is given in Fig. 7. It is possible with the aid of this figure, and accompanying electronmicrographs, to discuss and relate the major events in the chronological development of virus infection and replication, ML development and other cytopathic and/or structural and functional changes controlled by the virus.

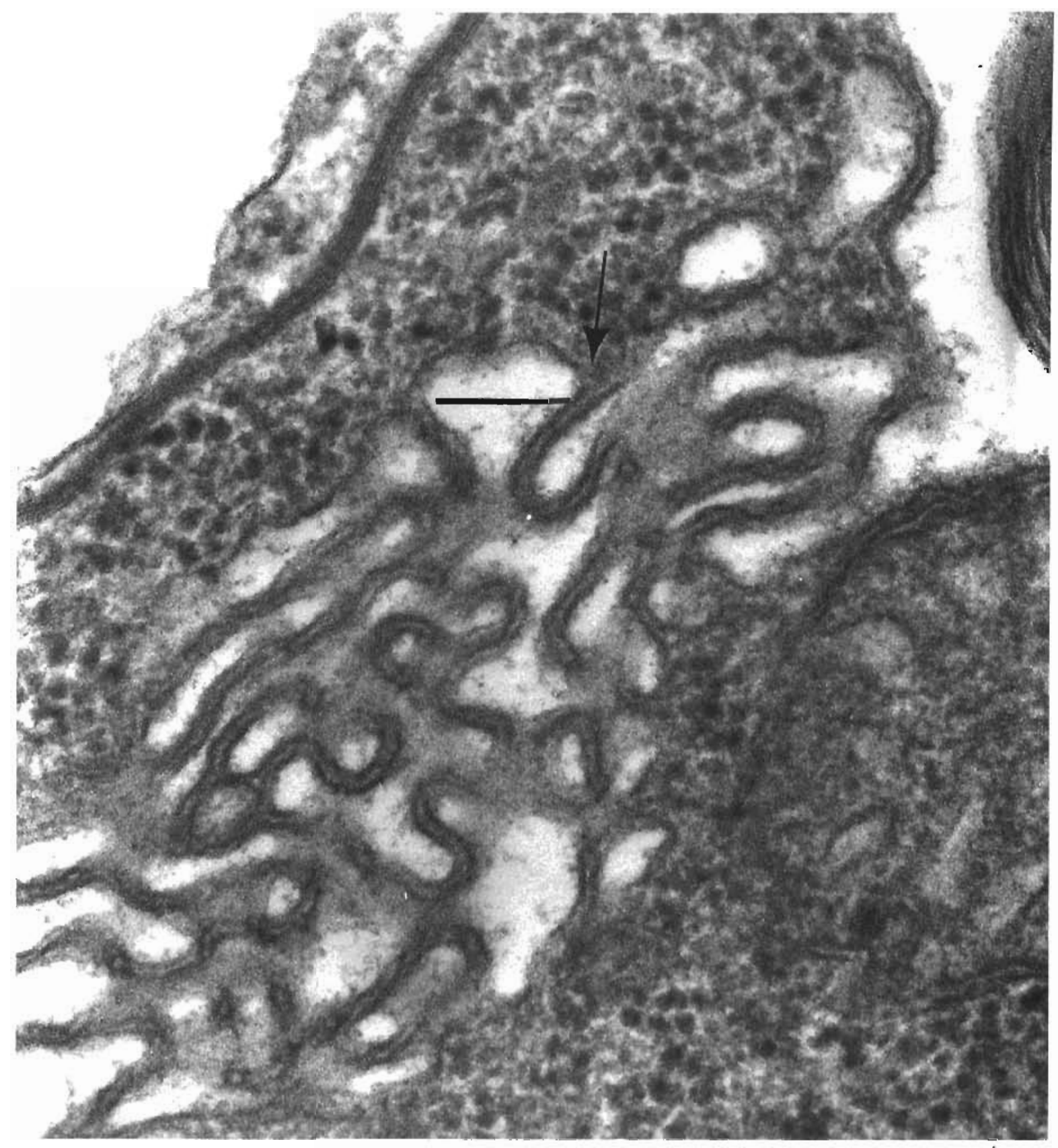

Fig. 6. Higher magnification of portion of ML probably having originated from Colgi; note fundamental structure of 2 closely opposed membranes (arrow) and many free ribosomes in adjacent cytoplasm; dark, amorphous, slightly granular material is found in cisternae fromed by ML membranes; unit membrane of cells plasmalemma at upper left for comparison with ML membranes. $\mathrm{Bar}=250 \mathrm{~nm}$ 
At the initiation of infection with $\mathrm{BP}$, host crustacean cells have typical cellular morphology, possessing features of most normal metazoan cells. BP infection and replication began with uptake and translocation of $\mathrm{BP}$ virions by host cells. The BP virion uncoated at the nuclear pore (Fig. 7A). Following presumed viral genome integration, a series of nuclear and cytoplasmic changes occurred. The most salient of these in order of occurrence were: margination of heterochromatin, appearance of granular and fibrillar virogenic stromata concurrent with out-folding of outer nuclear envelope membranes and dilation of Golgi and ER vesicles (Figs. 2, 5 and $7 \mathrm{~B}, \mathrm{C}$ ). ML initiation and early proliferation per se was characterized by dilations of the nuclear envelope, Golgi, and ER vesicles and distortions as illustrated in Figs. 2, 4, 5 and $t B$.

Concomitant with the early ML development, just described, was the detachment of polyribosomes from ER and appearance of many free ribosomes (monosomes) in the cytoplasm of infected cells (Figs. 1,5 and $7 \mathrm{~B}, \mathrm{C})$. Also, at this stage developing and complete nucleocapsids and membrane fragments were observed in infected cell nuclei, but occlusion body formation had not yet begun (Figs. 5 and $7 \mathrm{C}$ ). Mitochondria were either sequestered in one portion of the cell usually intimately associated with the $\mathrm{ML}$, or were largely absent in sections (Figs. 1, 7D, 9 and 10). Usually, at this stage there was a paucity of normal cytoplasmic organelles in the cell.

Assumed subsequent stages of ML development and enlargement apparently proceeded via tubular, concentric, vesicular, and labyrinthian proliferation of membranes concomitant with appearance and growth of occlusion bodies or polyhedra from crystallizations of 20 to $25 \mathrm{~nm}$ particles in the nucleus (Figs. 1, 7 A, B, 8, 9, 10 and 11 ). In later stages occlusion bodies were fully formed in their characteristic tetrahedral (triangular in thin section) shapes (Figs. 7D and 9), and the enlarged, infected nuciei also contained many cnvcloped free and occluded virions, and numerous free spherical particles $(20 \mathrm{~nm})$, but contained little or no heterochromatin (Figs. 3, 7D, 9 and 12).

The final stages of the virus reproductive cycle were characterized by the bizarre unfolding and loss of structural organization of the heretofore highly structured ML. These stages of infected cells/nuclei were found

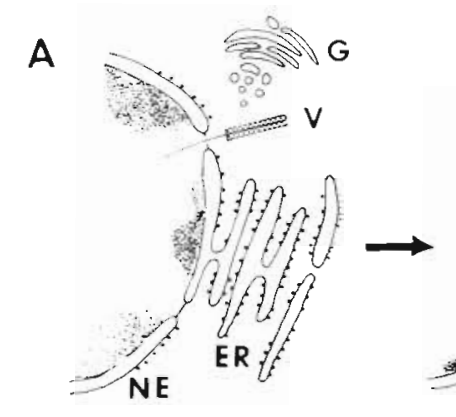

B

C
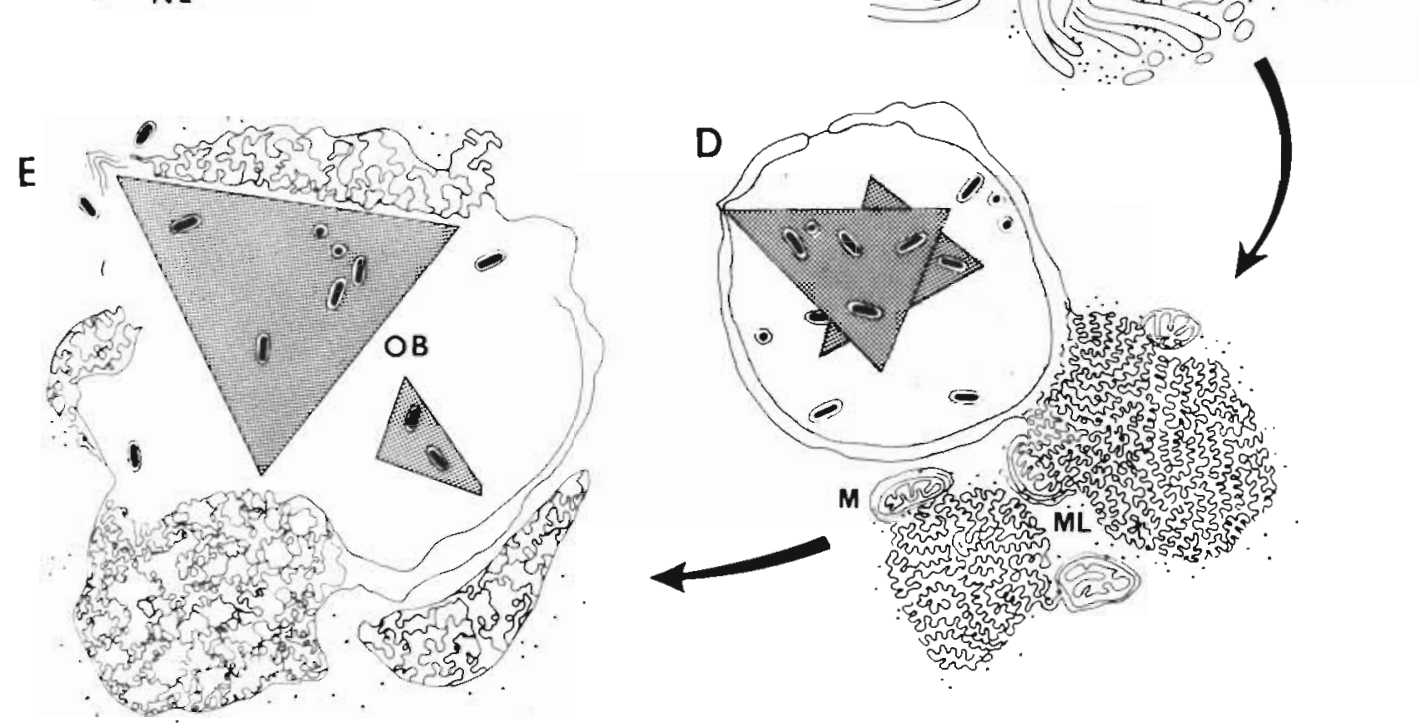

$62 \backslash G M P$

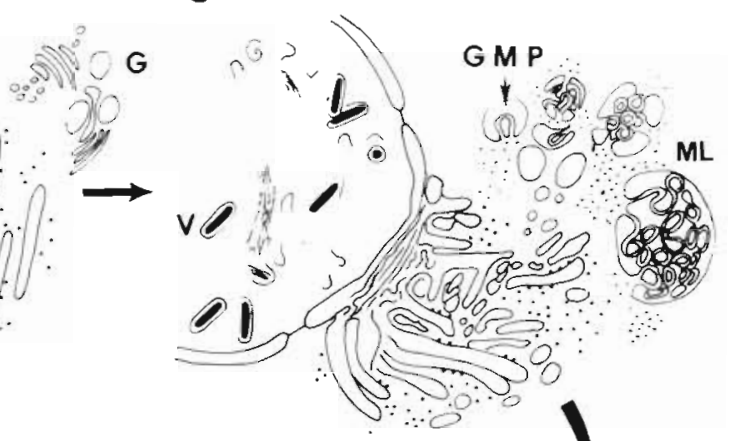

D

Fig. 7. Semi-diagrammatic depiction of presumed morphogenetic stages of origin and development of the ML; compare with stages described in Table 1 and in text. V: virion; G: Golgi; ER: endoplasmic reticulum; NE: nuclear envelope; R: ribosomes; GMP: early Golgi membrane proliferation. ML: membranous labyrinth; M: mitochondria; OB: occlusion body 
lysed or opened and occlusion bodies, free vinons, and all other inclusions were released from them (Figs. $7 \mathrm{E}$ and 13).

\section{DISCUSSION}

Hypothetical role of the ML in virus reproduction and fate

The ML appears in its labyrinthian form to be a highly ordered modification of the cytocavitary system (sensu Trump \& Arstila 1971) of BP-ınfected cells (Figs $1,7,9$ and 12). Its orıgin and morphogenesıs chronolog ically correspond with important steps in the production, assembly. and maturation of both BP virions and occlusion bodies or polyhedra (Table 1). Several lines of vicarious evidence for possible functional roles of the ML in BP reproductuve processes may be percelved in the preceding description of the ML. Subsequent discussion will examine these and additional pieces of evidence and postulate possible functions of the ML.

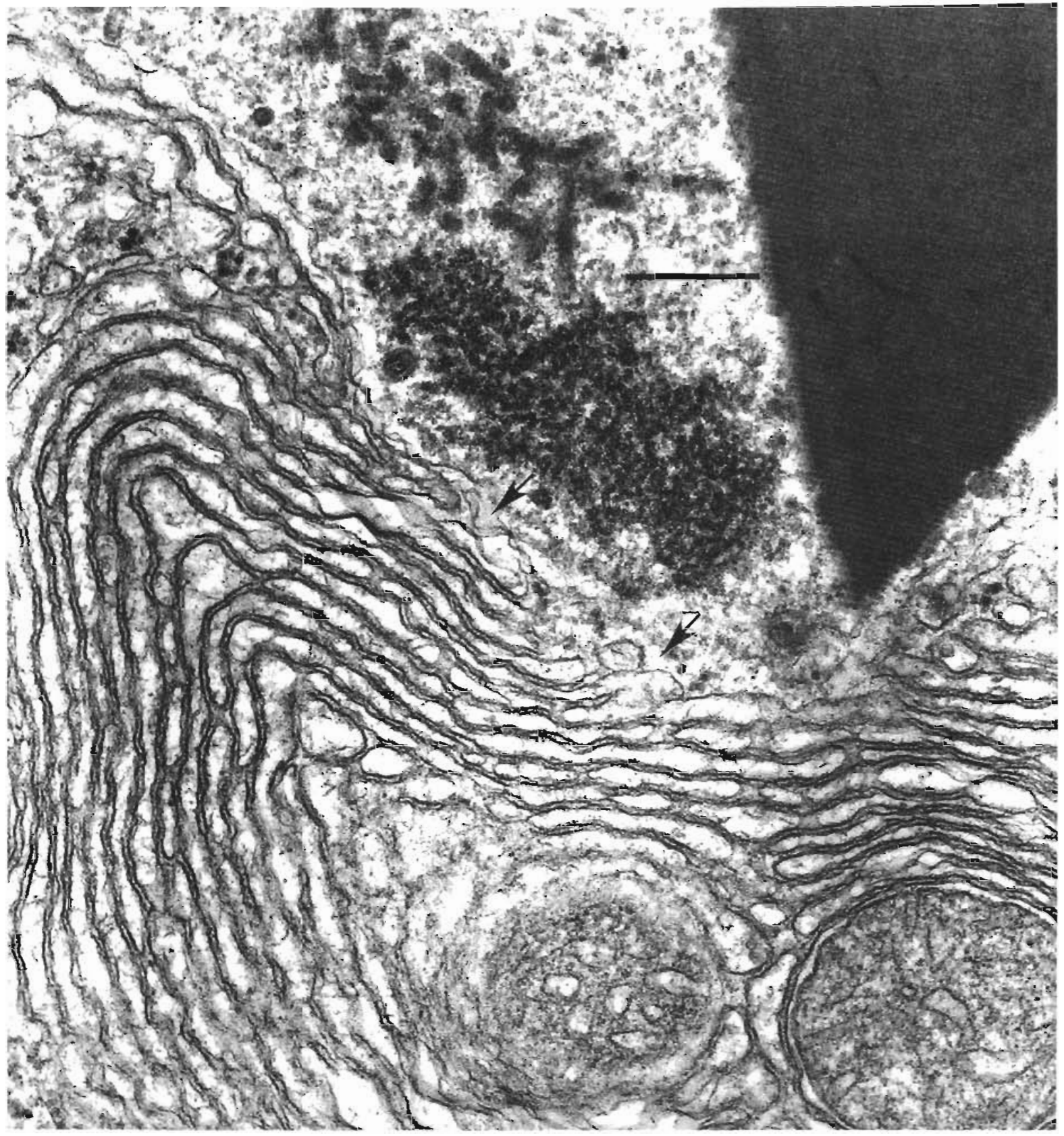

Fig. 8. Concentric form of ML demonstrating firm connection with outer and inner membranes of nuclear envelope (arrows); not occlusion of mitochondria in the ML, and intımate association of ML membranes and cistemae with outer membranes of mitochondrı Bar $=320 \mathrm{~nm}$ 
Another Baculovirus host cell system has more recently been described by Lightner \& Redman (1981) in reared Taiwanese post larval shrimp Penaeus monodon. This baculovirus, coded MBV (Monodon Baculovirus), also causes the formation of an ML in infected host cells. Though MBV is distinct from BP in many of its characteristics (OB form, size, etc.), the ML it causes in its hosts cells is remarkably similar to the ML described here for BP-infected crustacean cells. Lightner et al. (1983) described in some detail the form of the ML of MBV. One of their observations, pertinent to this paper's subject, is the cytoplasmic occurrence of small polyhedrin (protein constituting the occlusion bodies of baculoviruses) inclusions in association with vesicles of the Golgi and ER that are probably continuous with cisternae of the ML. Lightner et al. (1989) later observed the same phenomenon in BP-infected cells from Penaeus stylorostris (e.g. formation of BP occlusion bodies without virus, near ML in cytroplasm of infected cells). This observation supports all other data that indicate that the polyhedrin protein for both BP and MBV is synthesized at the ribosomes of the ER or by ribosomes (monosomes) in the cytoplasm near the ML and normally accumulates in or near the ER and

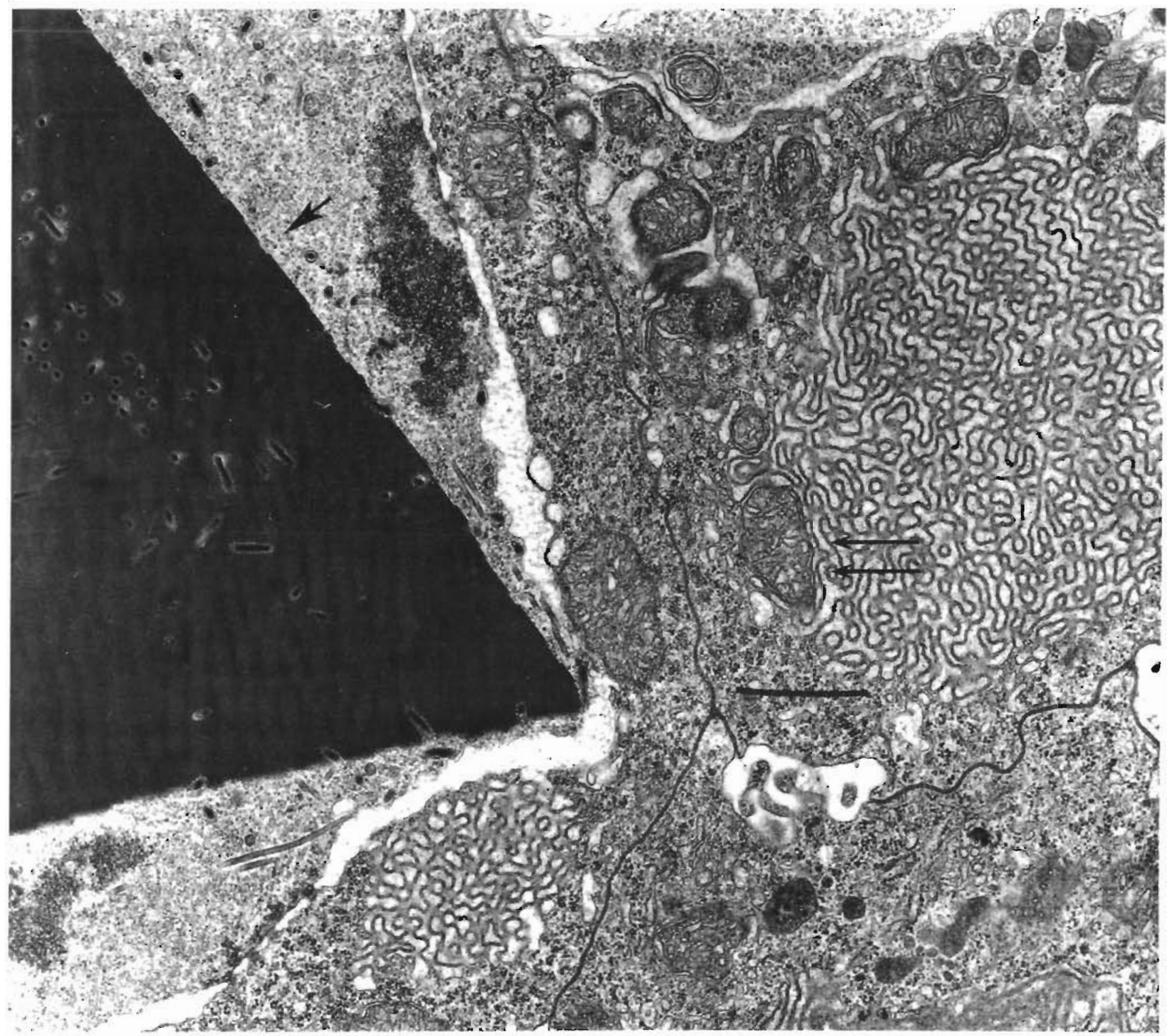

Fig. 9. Association of ML membranes and mitochondrion in an intimate fashon (arrows); note extended ML membrane that encircles nearby mitochondria; note also spherical particles (ca $20 \mathrm{~nm}$ ) in the nucleus of the other cell (single arrow) and their association with the growing plane of the paracrystalline OB; these particles are polyhedrin subunits lhat $\mathrm{f}(\mathrm{Irm}$ the $\mathrm{OB}$. Bar $=1$ um 
Golgi. The dark amorphous material in the ML custernae of BP-infected cells and the sphencal bodies (Fig 10) occasionally seen in the ML of BP may represent ribosomal synthesized material, and actually be viral structural precursors.

Along what path and how, then, may the precursor polyhedrin and other proteins be transported to the nucleus of the BP- and MBV-infected cells? I would hypothesize that, following integration, the baculovirus genetically controls the membrane-bound cytocavitary system of the host cell and directly or indirectly elicits formation of the ML via membrane prolnteration to provide a condunt or transport system (ML cisternae) for viral structural precursors transfer from site of synthesis in the cytoplasm (rough ER or cytoplasmic ribosomesmonosomes) to the nucleus where both virion and occlusion body assembly occurs Perhaps virion component precursor peptides are the main products transported or diffused to the nucleus via the ML cisternae and system of membranes it is known, for insect

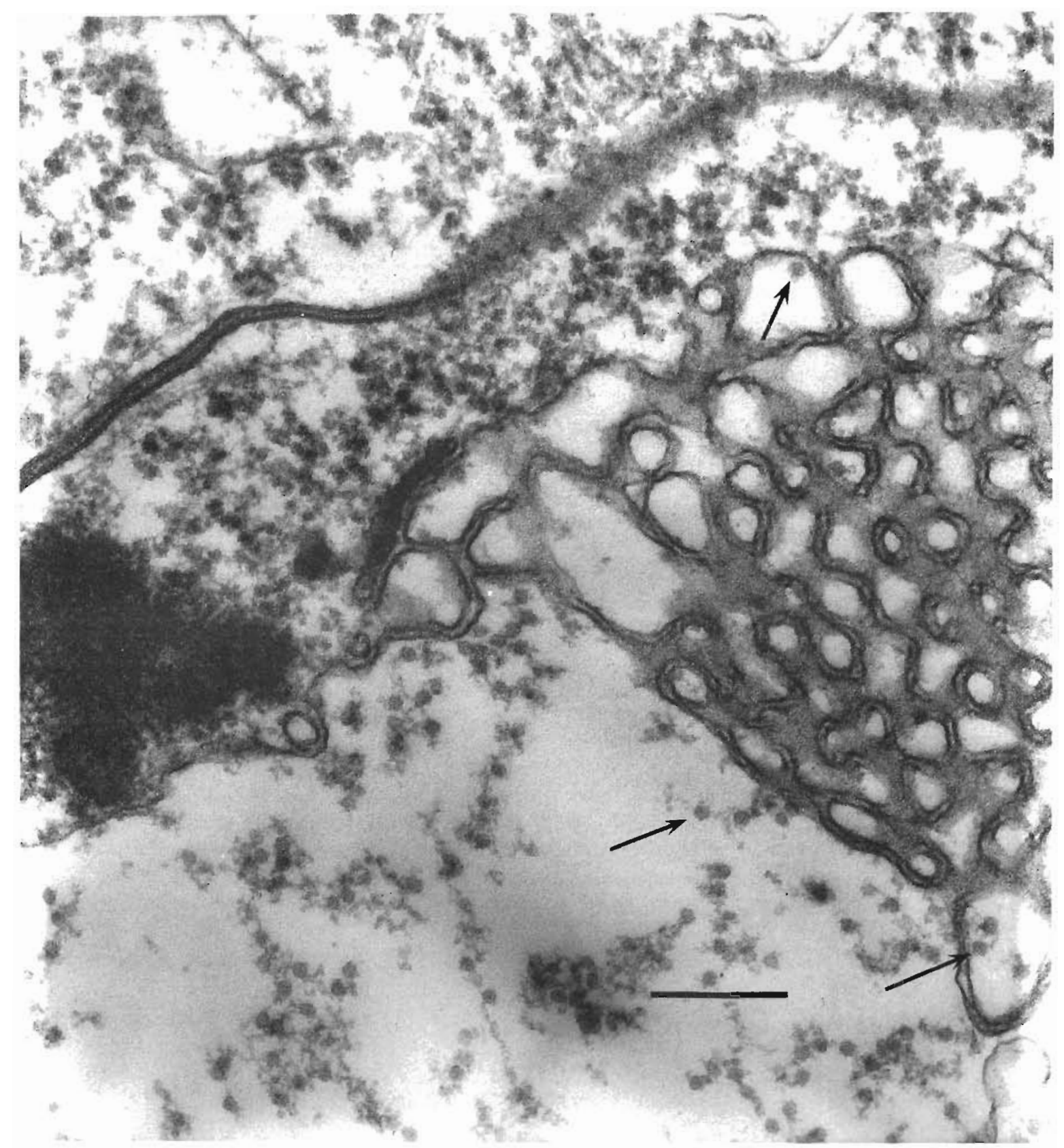

Fig. 10. Dark, granular, amorphous material and small, sphencal partıcles (ca $20 \mathrm{~nm}$ ) (arrows) simular to OB subunits in nucleus associated with ML membranes in cytoplasm of BP-infected cell Bar $=250 \mathrm{~nm}$ 
baculoviruses, that the polyhedrin synthesized at the ribosomes in the cytosol probably is targeted to the nucleus directly through the cytosol instead of through a membrane-bound system. We do not know exactly how polyhedrin is transported to the nucleus in the BPshrimp system. An additional, related fuction of the ML could be the provision of increased intracellular membrane surface area for membrane-bound enzymatic functions (such as ATPase activity related to releasing the energy from ATP required for transport of precursors and assembly of the virion and occlusion bodies in the nucleus, where there are no known protein synthetic mechanisms). Few known viruses compare with the occluded baculoviruses in programming their host cells to produce relatively large amounts of specialized virus components and huge amounts of polyhedrin for production of the largest known viral-related nuclear inclusion, the $O B$. These excessive demands for viral material alone could explain this dramatic modification of a cell's cytocavitary system, resulting in an exaggerated structure such as the ML that would provide a basis for transport and energy production.

The first piece of evidence vicariously suggesting a possible functional role of the ML in virus reproduction and/or $\mathrm{OB}$ production is the fact that the formation of the ML slightly precedes and occurs simultaneously with virogenesis and $\mathrm{OB}$ formation. The ML begins to form early during virus reproduction and usually can be found at maximum order and size during and immediately after $O B$ formation. In regard to this, many baculovirus-induced polypeptides have been identified in virus-infected insect cells (Kelly 1982). The induction of these peptides is time-sequenced. In insect baculovirus infected cells, polyhedrin is synthesized 12 to $15 \mathrm{~h}$ post infection, accumulates in the cytoplasm until about $18 \mathrm{~h}$ post infection, and is then slowly transferred to the nucleus where virus assembly and occlusion body formation occurs (Kelly 1982). Presently the exact time, post infection, required for virus assembly and $\mathrm{OB}$ formation in the BP crustacean cell system is not known, but from infectivity studies to date, it appears to be similar to the time required by the related insect bacuoviruses (a matter of hours). Therefore, the ML forms in time, in the BP-mediated system, to precede and be preseni duriny possible viral peptide synthesis and transport to the nucleus.

It is interesting to note that a time series of induction of proteins has been found for herpesviruses and iridoviruses (Elliot \& Kelly 1980), and that considerable modification of cytoplasmic membranes with cisternae (some changes similar to the ML of BP) have been associated with the herpesvirus reproduction in RL-33 cells (Morita \& Iida 1980). Still other reports for a variety of viruses indicate the formation of cytoplasmic, membranous structures, such as annulate lamellae, multivesicular bodies, and concentric membrane masses from ER, outer nuclear envelope and other organelles (Kim \& Boatman 1967, Merkow et al. 1970, Patrizi \& Middle-

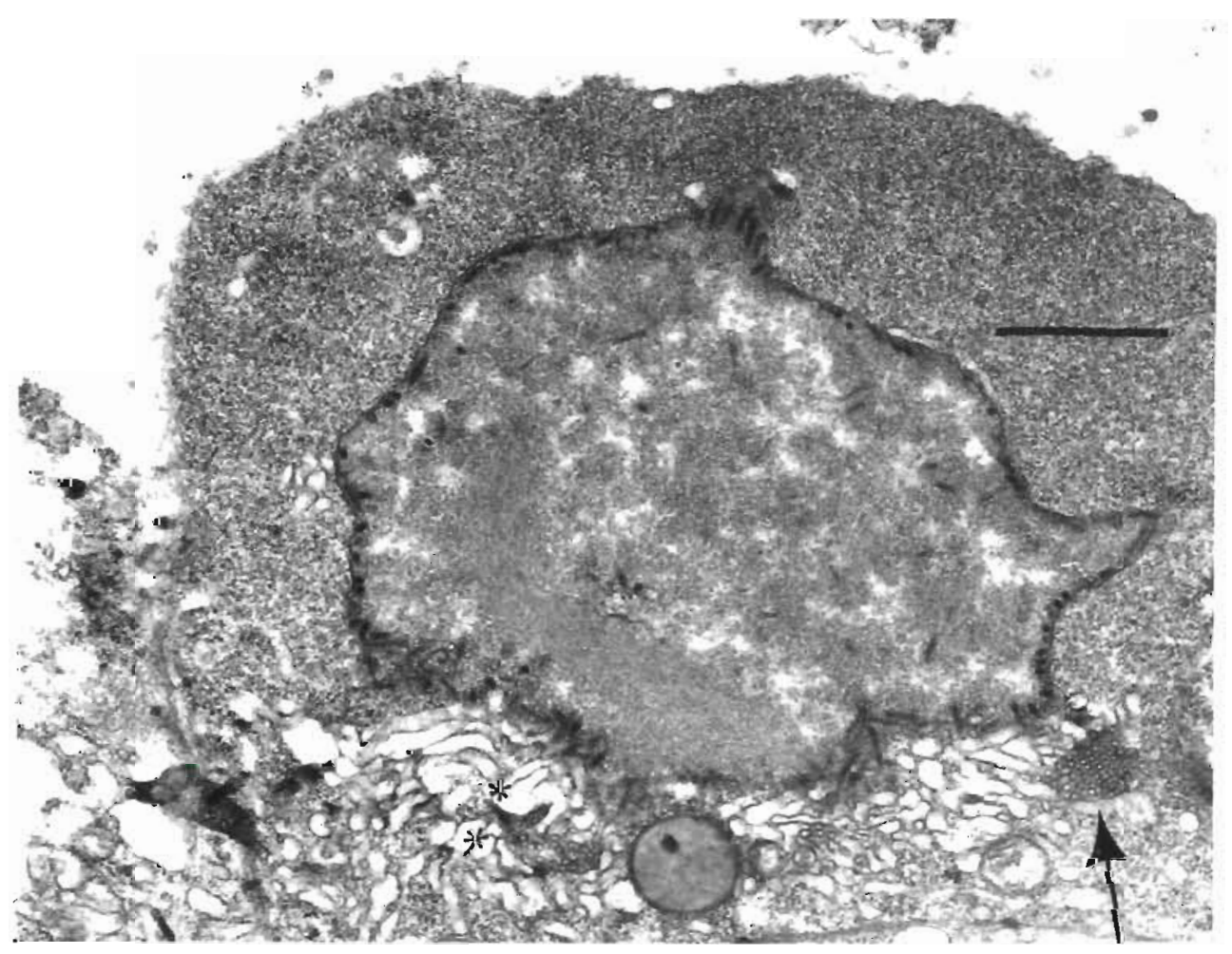

Fig. 11. Growth and fusion of early tubular $(*)$ and labyrinthian forms (arrows) of membrane complexes in Stage $\mathrm{C}$ of development; note abundance of free ribosomes in cytoplasm. Bar $=2$ um 
kamp 1970, Russo et al. 1987); usually, these authors did not suggest functions other than aber ant virus assembly processes for these membranous structures

The derivation of the ML, in BP-crustacean cell systems, from the very flexible Golg1, outer nuclear envelope and ER, all part of the cytocavitary system of the cell (Trump \& Arstila 1971, Porter \& Bonneville 1968), and the known role of this membrane-bound system in cells, suggests that viruses could turn this role of packaging and transporting proten and complex carbohydrates to their own needs in reproduction. If this were the case in the ML, then the direction of flow of these protens and perhaps other precursor substance would be (under virus control) largely reversed toward the nucleus where nuclear, DNA virus assembly and occlusion body formation occurs, rather than toward the plasma membrane where normal secretion of synthesızed, cellular products occurs. Such an active transport phenomenon would, of course be energy demandıng. In regard to the earlier postulated ATPase energy

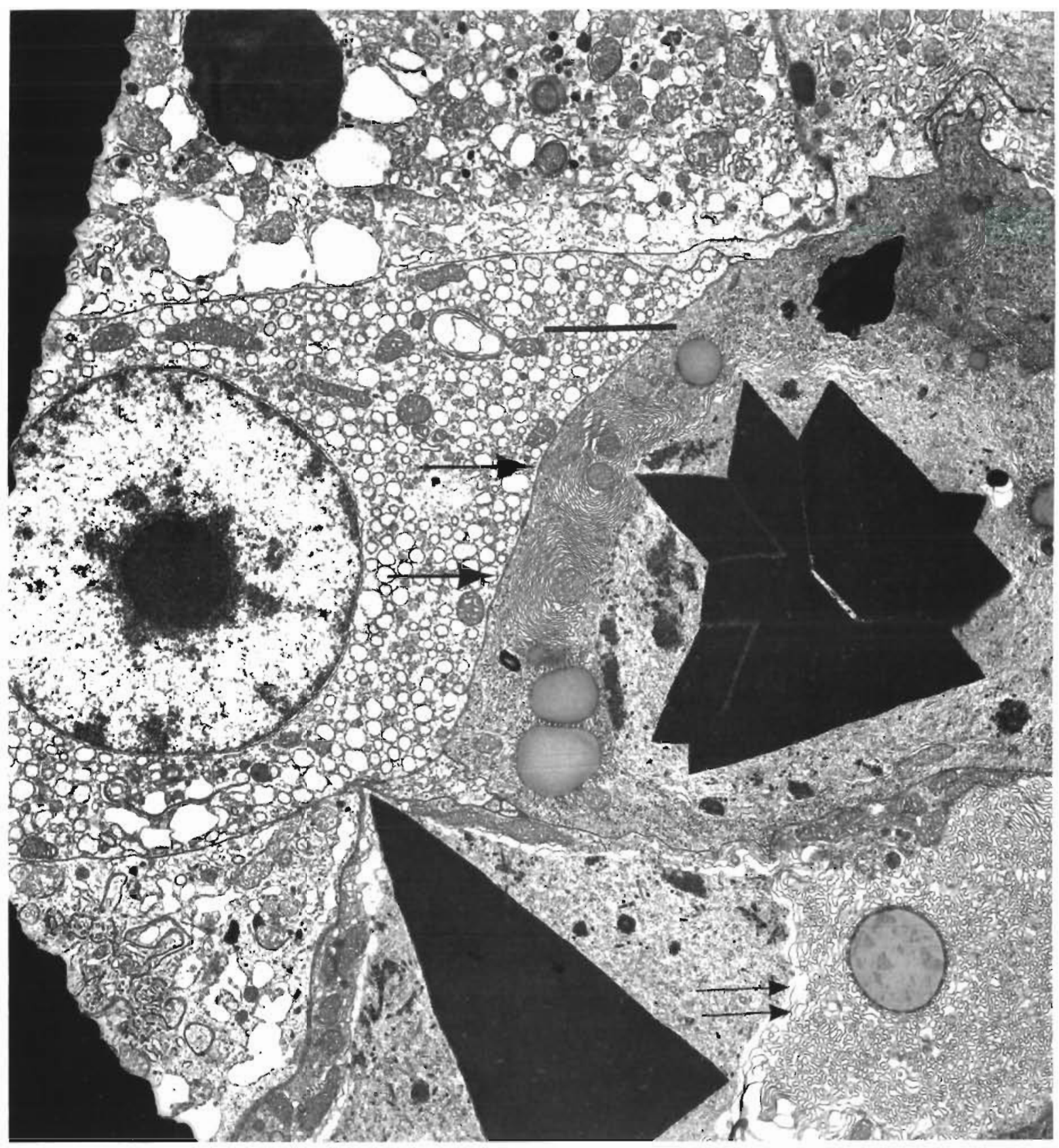

Fig. 12. Highly organized structure of the ML in Stage D. BP-infected cells; note the extensive areas filled by the ML in both concentric form (single arrows) and labyrinthian form (double arrows), triangular forms of the mature occlusion bodies are apparent, as are occluded, rod-shaped virions. Bar $=4 \mu \mathrm{m}$ 
functions, it is interesting to note that the remaininic mitochondria in BP-infected cells, more often than not are closely associated with the ML complex (Figs. 1, 3 , $7 \mathrm{D}, 8$ and 9). Thus, they could supply the ATP necessary for energy to transport substances or for assembly/ synthesis processes. Membranes from the ML have been observed to encircle or occlude proximal mitochondria (Figs. 1, 3, 7D, 8 and 9), thus providing a direct or intimate path for mitochondrial generated ATP to possible ML membrane-bound ATPase.
Another ultrastructural observation that supports a reason-to-be status for the $\mathrm{ML}$ is that in middle to late stages of BP infections there remains very little or no normal ER or Golgi in infected cells, yet the ML is at a full size and order and is maintdined as an intact entity in these stages (Figs, 1, 9 and 12). The possibility exists that at these times in BP-infected cells, the remaining portion of the cytocavitary system (Golgi, ER) for carrying out virally directed functions is in the form of the highly modified ML

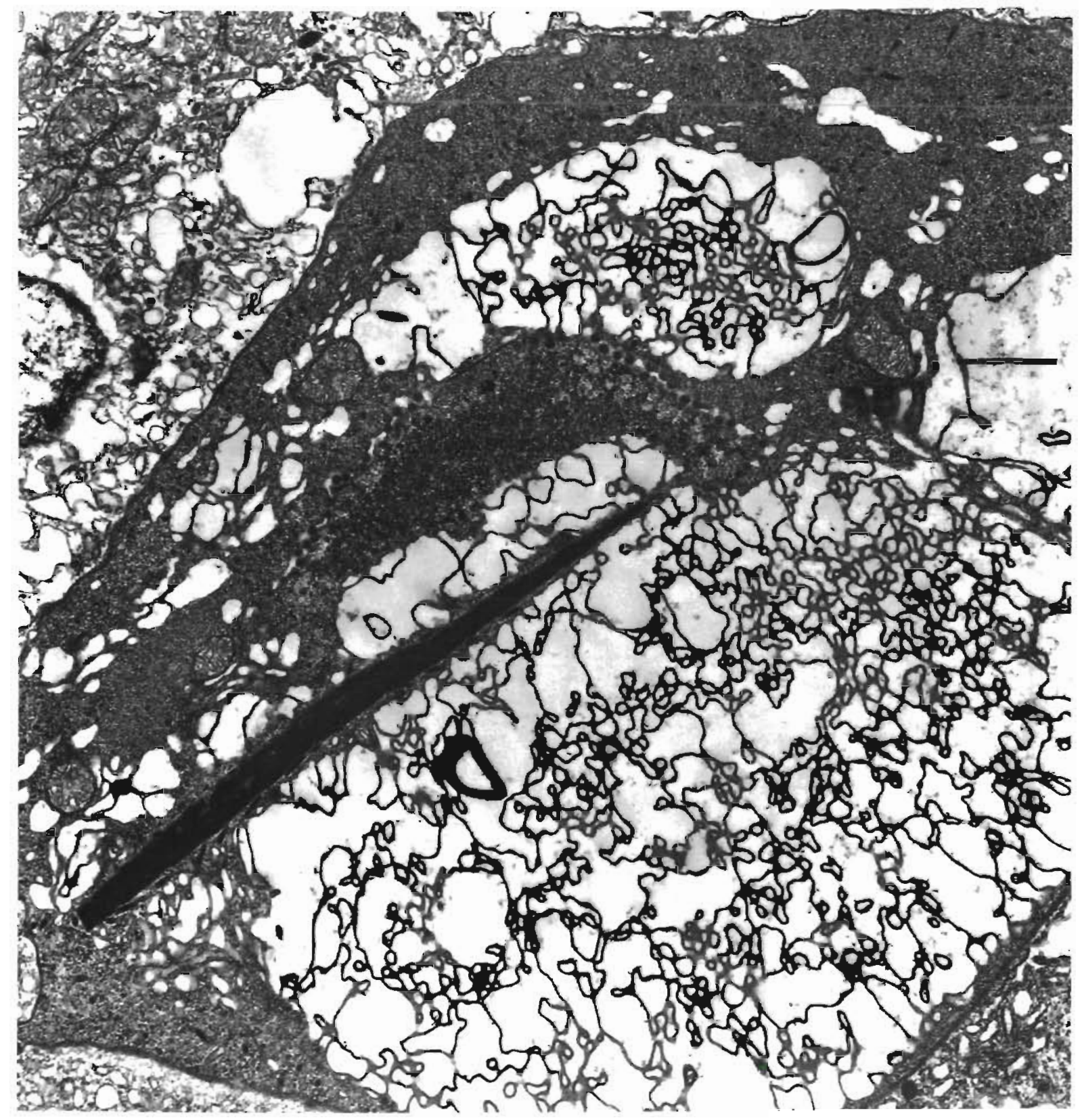

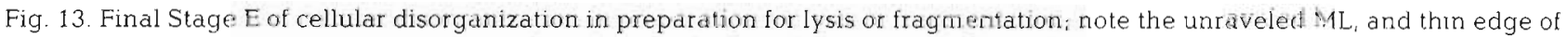
an $O B$ in disorganized nuclear remnant. $\mathrm{Bar}=1 \mathrm{um}$ 
A final role for the ML in BP-infected cells seems to be a degenerative one. In order for the OB's and virions remaining in the nucleus to be freed the cell's structural integrity must be breached. This is partly achieved by the grow th of the $O B$ which, if great enough, will rupture nuclear and cell membranes (Fig 7E). At a late point in the viral reproductive cycle, however, the ML begins to unravel, becomes disorganized, and because of its large mass in the cytoplasm, contributes to the eventual collapse of the whole cell (Figs. 7E and 13), freeing OB's and virions into the intercellular spaces. This provides for dissemination of the viral progeny in both protected (occluded form) and in free form.

\section{Possible methods for experimentally demonstrating the postulated ML functions and implications of possible functions}

Unfortunately there are no permanent crustacean cell lines with which to carry out experimental BP infections. Any experimental demonstration of a role for the ML in those shrimp digestive gland cells that permit growth of $\mathrm{BP}$ would have to be conducted with whole shrimp hosts (adult or early metamorphic stages). Insect cells, readily infected by insect baculoviruses, are culturable and amenable to experimentation in vitro. Membrane proliferations occur in granulosis virus-infected cells of the insect Trichoplusia ni (Summers 1971) and have some resemblances to the ML in crustacean cells, but usually are not as massive or as extensive.

With amino acid pulse-labeling, via feeding or injection of labeled amino acids characteristic of occlusion body polyhdrin, one may be able to track the transport of the amino acids through the ML utilizing ultrastructural autoradiography. Harvest of the ML as a discrete cellular component by cell component separation (density gradients and ultracentrifugation) from previously infected shrimp might yield enough material to determine presence of certain peptides and/or proteins in ML-containing cell fractions. Other contemporary immunological, enzyme histochemical or molecular probes might be used to determine content of the ML. If certain proteins are heavily associated with the ML, further correlations with ML stages and viral reproduction and assembly in situ could be attempted.

The current study provides vicarious, suggestive evidence that the ML produced in all BP-infected cells may not be merely a degenerative cytopathic effect, but may represent a viral-directed, highly organized modification of the host cell's own internal membrane systems that is enlisted to essentially aid in viral and perhaps, $O B$ assembly, and eventual viral dissemination

Responsible Subject Editor- Dr J. E Stewart, Halifax, N.S. Canada
Perhaps the ML in BP-infected shrimp cells is an unusually dramatic, exaggerated, morphologic expression of what occurs more subtly in other nuclear, DNA virus/host cell systems in regard to transport of virus products between cell compartments. If this is so, then the BP/crustacean (virus/host) system could serve as an investigative model with which to study these processes.

\section{LITERATURE CITED}

Couch, J. A (1974). An enzootic nuclear polyhedrosis virus of pink shrimp: ultrastructure, prevalence, and enhancement. J. Invert. Pathol. 24: 311-331

Couch, J. A. (1989). Inclusion body viruses. II. Baculoviruses of invertebrates other than insects. CRC Press (in press)

Elliot, R. M., Kelly, D. C. (1980). Frog virus 3 replication: induction and intracellular distribution of polypetides in infected cells. J. Virol. 33: 28-51

Hayat, M. A., Giaquinta, R. (1970). Rapid fixation and embedding for electron microscopy. Tissue Cell 2: 191-195

Kelly, D. C. (1982). Baculovirus replication. J. gen. Virol. 63: 1-13

Kim, K. S. W., Boatman, E. S. (1967). Electron microscopy of monkey kidney cell cultures infected with rubella virus. J. Virol. 1: 205-214

Lightner, D. V., Redman, R. M. (1981). A baculovirus-caused disease of the penaeid shrimp, Penaeus monodon: J. Invert. Pathol. 38: 299--302

Lightner, D. V., Redman, R. M., Bell, T A. (1983). Observations on the geographic distribution, pathogenesis, and morphology of the baculovirus from Penaeus monodon Fabricius. Aquaculture 32: 209-233

Lightner, D. Y., Redman, R. M., Ruiz, A. E. A. (1989). Baculovirus penaei in Penaeus stylirostris (Crustacea:Decapoda) cultured in Mexico: unique cytopathology and a new geographic record. J. Invert. Pathol. 53: 137-139

Matthews, R. E. F. (1982). Classification and nomenclature of viruses. Intervirology 17: 1-199

Merkow, L. P., Slifkin, M., Pardo, M., Rapoza, N. D. (1970). Pathogenesis of oncogenic adenoviruses. VII. The origin of annulate lamellae in LLC-M $\mathrm{K}_{2}$ cells infected with SC-30. J. ultrastruct. Res. 30: 344-353

Morita, M. Iida, T (1980). Morphological observations of the replication of Herpesvirus tamarinus in RL-33 cells. Arch. Virology 66: 53-65

Patrizi, G., Middlekamp, J. N. (1970). Development and changes of annulate lamellae complexes in rubella virusinfected RL-13 cells. J. ultrastruct. Res. $31407-423$

Penman, S. (1986). Virus metabolism and cellular architecture. In: Fields, B. N., et al. (eds.) Fundamental virology. Raven Press, New York, p. 169-182

Porter, R., Bonneville, M. A. (1968). Fine structure of cells and tissues 3rd edn. Lea and Febiger, Philadelphia

Russo, M., DiFranco, A., Martelli, G. P. (1987). Cytopathology in the identification and classification of Tombusviruses. Intervirology 28: 134-143

Summers, M. D. (1971). Electron microscopic observations on granulosis virus, entry, uncoating, and replication processes during infection of the midgut cells of Trichoplusia ni. J. ultrastruct. Res. 35: 606-625

Trump, B. F. Arstila, A. (1971). Cell injury and cell death. In: LaVia, M., Hill, R. B. (eds.) Principles of pathobiology. Oxford Univ. Press, New York, p. 9-95

Manuscript first received: March 8, 1989

Revised version accepted: June 27, 1989 